\title{
Peristaltic transport of viscoelastic bio-fluids with fractional derivative models
}

\author{
Emilia Bazhlekova, Ivan Bazhlekov \\ Institute of Mathematics and Informatics \\ Bulgarian Acad. Sci., Sofia, Bulgaria \\ e.bazhlekova@math.bas.bg, i.bazhlekov@math.bas.bg
}

Received: 28 October 2015, accepted: 16 May 2016, published: 26 May 2016

\begin{abstract}
Peristaltic flow of viscoelastic fluid through a uniform channel is considered under the assumptions of long wavelength and low Reynolds number. The fractional Oldroyd-B constitutive viscoelastic law is employed. Based on models for peristaltic viscoelastic flows given in a series of papers by Tripathi et al. (e.g. Appl Math Comput. 215 (2010) 3645-3654; Math Biosci. 233 (2011) 9097) we present a detailed analytical and numerical study of the evolution in time of the pressure gradient across one wavelength. An analytical expression for the pressure gradient is obtained in terms of Mittag-Leffler functions and its behavior is analyzed. For numerical computation the fractional Adams method is used. The influence of the different material parameters is discussed, as well as constraints on the parameters under which the model is physically meaningful.
\end{abstract}

Keywords-Riemann-Liouville fractional derivative; Mittag-Leffler function; viscoelastic flow; fractional Oldroyd-B constitutive model; peristalsis.

\section{INTRODUCTION}

Recently, Fractional Calculus has gained considerable popularity mainly due to its numerous applications in diverse fields of science and engineering. Fractional Calculus allows integration and differentiation of arbitrary order, not necessarily integer. More precisely, it deals with integro- differential operators, where the integrals are of convolution type with weakly singular power-law kernels.

Extensive applications of Fractional Calculus can be found in the constitutive modeling of viscoelasticity, see [3], [4], [10], [18], [20] and the references cited there. The fractional order constitutive models (proposed in the beginning in an implicit way, see for a historical overview [19], [22], [29]) appear to be a valuable tool for describing viscoelastic properties. Unlike the classical models which exhibit exponential relaxation, the models of fractional order show power-law behavior which is widely observed in a variety of experiments. They provide a higher level of adequacy preserving linearity and give the possibility for relatively simple description of the complex behavior of non-Newtonian viscoelastic fluids.

The generalized fractional Oldroyd-B constitutive law belongs to the class of linear fractional models of viscoelastic fluids. It is obtained by replacing the first order derivatives in the classical Oldroyd-B model by derivatives of fractional order. The corresponding constitutive equation in the one-dimensional case is given by

$$
\left(1+\lambda_{1}^{\alpha} D_{t}^{\alpha}\right) \tau(t)=\eta\left(1+\lambda_{2}^{\beta} D_{t}^{\beta}\right) \dot{\varepsilon}(t),
$$

where $\tau(t)$ is the shear stress, $\varepsilon(t)$ - shear strain, 
the over-dot denotes the first time derivative, $\eta>0$ is the dynamic viscosity of the fluid, $\lambda_{1}, \lambda_{2} \geq 0$ are parameters related to the relaxation and retardation times, respectively, and $D_{t}^{\alpha}$ and $D_{t}^{\beta}$ are fractional Riemann-Liouville derivatives in time of orders $\alpha$ and $\beta$, where $0<\alpha \leq 1,0<\beta \leq 1$. The generalized Oldroyd-B model (1) encompasses a large class of fluids: Newtonian fluid $\left(\lambda_{1}=\lambda_{2}=0\right)$, fractional second grade fluid $\left(\lambda_{1}=0, \lambda_{2}>0\right)$, fractional Maxwell fluid $\left(\lambda_{2}=0, \lambda_{1}>0\right)$. In [23] a very good fit with experimental data is achieved for the fractional Oldroyd-B model. Unidirectional flows of viscoelastic fluids with the fractional Oldroyd-B constitutive law are studied in [5], [11], [13], [17], [21], to mention only few of many recent publications.

The transportation of many biophysical fluids is controlled by a special mechanism called peristalsis. The mechanism includes involuntary periodic contraction followed by relaxation and expansion of the ducts through which the fluids pass. It is inducted by the propagation of electrochemically generated waves along the vessels containing fluids. Examples from physiology where the peristaltic transport is prevalent are the movement of chyme in the small intestine, transport of bile in bile ducts, transport of lymph in the lymphatic vessels, etc. The complex physical nature of peristaltic flows of non-Newtonian fluids stimulated significant attention in the applied mathematics and engineering sciences research communities. For recent research on this topic we refer to [1], [2], [8], [9], [15], [28].

Fractional derivative models for peristaltic transport of viscoelastic fluids are derived in a series of papers by Tripathi et al. (e.g. [24], [25], [26], [27]) As noted in [26] such models are appropriate for describing the chyme movement in the small intestine, by considering the gastric chyme as a viscoelastic fluid. In [26] and [27] peristaltic transport through a cylindrical tube of fractional Oldroyd-B fluid is studied, with $0<\alpha \leq \beta \leq 1$. In [26] inclined tube is considered and in [27] wall slip conditions are assumed. In [24], [25] the particular case of a fractional Maxwell model $\left(\lambda_{2}=0\right)$ is considered. For the numerical computations the Adomian decomposition and homotopy analysis methods are used in the aforementioned articles.

In the present work, peristaltic flow of viscoelastic fluid through a uniform channel is considered under the assumptions of long wavelength and low Reynolds number. The viscoelastic properties of the fluid are governed by the fractional Oldroyd-B constitutive equation. We employ the model proposed in [24] for the particular case of fractional Maxwell fluid $\left(\lambda_{2}=0\right)$ and generalize it in a straightforward way to cover also the case $\lambda_{2} \neq 0$. Since the considered model is non-stationary in nature and contains parameters which are timerelated such as the orders of the fractional time derivatives $\alpha$ and $\beta$, the relaxation and retardation times $\lambda_{1}, \lambda_{2}$, it is natural to study the time evolution of the physical quantities described by the model and the influence of the different parameters on this evolution.

Our main contribution is a detailed analytical and numerical analysis of the time evolution of the pressure gradient across one wavelength in the peristaltic flow. To the best of our knowledge, this issue has not been discussed before in the general case $\lambda_{2} \neq 0$. An explicit expression for the pressure gradient in terms of the Mittag-Leffler functions is derived and its behavior is studied. For the numerical computations a technique based on the fractional Adams method [6], [7] is used. Results of several numerical examples are given and the influence of the different material parameters is discussed as well as constraints on the parameters under which the model is physically meaningful.

The rest of the paper is organized as follows. In Section II the equation for the pressure gradient is derived. In Section III an analytical representation for the pressure gradient is obtained in terms of Mittag-Leffler functions and its behavior is analyzed. In Section IV the numerical method used for the computations is described. The obtained numerical results are given in Section $\mathrm{V}$ and the influence of the different material parameters is discussed. Section VI contains conclusions. Some 
basic definitions and results from Fractional Calculus which are used in this work are summarized in an Appendix.

\section{MATHEMATiCAL MODEL}

In this section we derive the equation for the pressure gradient in a peristaltic flow of a viscoelastic fluid with fractional Oldroyd-B constitutive model. This equation was originally proposed in [24] for the case $\lambda_{2}=0$. The necessary generalizations to the case $\lambda_{2} \neq 0$ are straightforward. Here we give for completeness the main steps in the derivation. For further details we refer to [24] and the related works [17], [21], [25], [26], [27].

The fundamental equations governing the unsteady motion of an incompressible viscoelastic fluid are the continuity equation:

$$
\nabla \cdot \mathbf{V}=0
$$

and the general Cauchy momentum equation:

$$
\rho\left(\frac{\partial \mathbf{V}}{\partial t}+(\mathbf{V} \cdot \nabla) \mathbf{V}\right)=\nabla \cdot \boldsymbol{\sigma}
$$

where $\mathbf{V}$ is the velocity vector, $\rho$ - fluid density, $\boldsymbol{\sigma}$ - Cauchy stress tensor:

$$
\boldsymbol{\sigma}=-p \mathbf{I}+\boldsymbol{\tau}
$$

where $p$ is the pressure and $\tau$ is the shear stress tensor, which for a viscoelastic fluid with the generalized fractional Oldroyd-B model satisfies the equation

$$
\left(1+\lambda_{1}^{\alpha} \frac{D^{\alpha}}{D t^{\alpha}}\right) \boldsymbol{\tau}=\eta\left(1+\lambda_{2}^{\beta} \frac{D^{\beta}}{D t^{\beta}}\right) \mathbf{A}_{\mathbf{1}} .
$$

Here $\eta>0$ is the dynamic viscosity of the fluid, $\lambda_{1}$ and $\lambda_{2}$ are parameters related to relaxation and retardation times, respectively, satisfying (see [23])

$$
\lambda_{1} \geq \lambda_{2} \geq 0
$$

$\alpha$ and $\beta$ are fractional parameters,

$$
0<\alpha \leq 1,0<\beta \leq 1,
$$

$\mathbf{A}_{\mathbf{1}}$ is the first Rivlin-Ericksen tensor given by

$$
\mathbf{A}_{\mathbf{1}}=\nabla \mathbf{V}+(\nabla \mathbf{V})^{T}
$$

and $\frac{D^{\gamma}}{D t^{\gamma}}$ denotes the upper convected time derivative

$$
\frac{D^{\gamma} \boldsymbol{\tau}}{D t^{\gamma}}=D_{t}^{\gamma} \boldsymbol{\tau}+(\mathbf{V} \cdot \nabla) \boldsymbol{\tau}-(\nabla \mathbf{V}) \cdot \boldsymbol{\tau}-\boldsymbol{\tau} \cdot(\nabla \mathbf{V})^{T}
$$

where $D_{t}^{\gamma}$ is the Riemann-Liouville fractional derivative, see (51) in the Appendix for the definition.

It is assumed that the relevant Reynolds number is small enough for inertial effects to be negligible and the wavelength to diameter ratio is large enough for the pressure to be considered uniform over the cross-section of the channel. As in [24] we consider a uniform horizontal two-dimensional channel with $h$ being the transverse displacement of the walls. Denote by $x$ the axis along the channel and by $y$ the transverse coordinate. Let $u$ be the velocity of the flow in the direction of the channel.

First, the above equations are rewritten in dimensionless form (for details see [24]). Here, for simplicity we keep the same notations. According to the assumption of low Reynolds number, one obtains inserting (3) in the momentum equation (2):

$$
\begin{aligned}
& \frac{\partial p}{\partial x}=\frac{\partial \tau_{x y}}{\partial y} \\
& \frac{\partial p}{\partial y}=0
\end{aligned}
$$

On the other hand, the constitutive equation (4) gives:

$$
\left(1+\lambda_{1}^{\alpha} D_{t}^{\alpha}\right) \tau_{x y}=\left(1+\lambda_{2}^{\beta} D_{t}^{\beta}\right) \frac{\partial u}{\partial y} .
$$

Applying the operator $\left(1+\lambda_{1}^{\alpha} D_{t}^{\alpha}\right)$ to both sides of (8) the following identity is deduced:

$$
\left(1+\lambda_{1}^{\alpha} D_{t}^{\alpha}\right) \frac{\partial p}{\partial x}=\left(1+\lambda_{1}^{\alpha} D_{t}^{\alpha}\right) \frac{\partial \tau_{x y}}{\partial y}
$$

Differentiating with respect to $y$ both sides of equation 10 one gets

$$
\left(1+\lambda_{1}^{\alpha} D_{t}^{\alpha}\right) \frac{\partial \tau_{x y}}{\partial y}=\left(1+\lambda_{2}^{\beta} D_{t}^{\beta}\right) \frac{\partial^{2} u}{\partial y^{2}} .
$$


E. Bazhlekova et al., Peristaltic transport of viscoelastic bio-fluids ...

From (11) and (12) one deduces the following equation for the pressure gradient $\frac{\partial p}{\partial x}$

$$
\left(1+\lambda_{1}^{\alpha} D_{t}^{\alpha}\right) \frac{\partial p}{\partial x}=\left(1+\lambda_{2}^{\beta} D_{t}^{\beta}\right) \frac{\partial^{2} u}{\partial y^{2}} .
$$

Boundary conditions for the velocity are given by

$$
\left.\frac{\partial u}{\partial y}\right|_{y=0}=0,\left.u\right|_{y=h}=0 .
$$

In the following integrations we essentially use the fact that the pressure does not depend on the transverse coordinate $y$, see 9 , i.e. $p=p(x, t)$. Integrating Eq. (13) with respect to $y$ twice and using boundary conditions (14) one obtains successively

$$
\begin{aligned}
& \left(1+\lambda_{1}^{\alpha} D_{t}^{\alpha}\right) \frac{\partial p}{\partial x} y=\left(1+\lambda_{2}^{\beta} D_{t}^{\beta}\right) \frac{\partial u}{\partial y} . \\
& \frac{y^{2}-h^{2}}{2}\left(1+\lambda_{1}^{\alpha} D_{t}^{\alpha}\right) \frac{\partial p}{\partial x}=\left(1+\lambda_{2}^{\beta} D_{t}^{\beta}\right) u .
\end{aligned}
$$

Denote by $Q$ the volumetric flow rate $Q=$ $\int_{0}^{h} u \mathrm{~d} y$. Then after one more integration 16 implies

$$
-\frac{h^{3}}{3}\left(1+\lambda_{1}^{\alpha} D_{t}^{\alpha}\right) \frac{\partial p}{\partial x}=\left(1+\lambda_{2}^{\beta} D_{t}^{\beta}\right) Q .
$$

Following [24] it is assumed that the wall of the channel undergoes contraction and relaxation given by

$$
h=1-\phi \cos ^{2}(\pi x),
$$

where $\phi$ is the amplitude of the wave. The transformations between the wave and the laboratory frames (an established procedure in peristaltic fluid dynamics, see [14]) are given in dimensionless form by

$$
X=x-t, Y=y, U=u-1, \theta=Q-h .
$$

Let $\bar{Q}$ denotes the averaged volumetric flow rate

$$
\bar{Q}=\int_{0}^{1} Q \mathrm{~d} t .
$$

Using the following relation from [14]

$$
\bar{Q}=\theta+1-\frac{\phi}{2}=Q-h+1-\frac{\phi}{2},
$$

Eq. (17) gives

$$
\left(1+\lambda_{1}^{\alpha} D_{t}^{\alpha}\right) \frac{\partial p}{\partial x}=\left(1+\lambda_{2}^{\beta} D_{t}^{\beta}\right) A,
$$

where

$$
A=-\frac{3}{h^{3}}(\bar{Q}+h-1+\phi / 2) .
$$

For further details on this derivation we refer to [24].

Let us emphasize that the function $A$ defined by (23) does not depend on time, but it depends on the spatial variable $x$ via the peristaltic wave parameters $h, \phi$ and $\bar{Q}$. Therefore we write $A=$ $A(x)$.

\section{ANALYTICAL PROPERTIES OF THE PRESSURE GRADIENT FUNCTION}

Since $A(x)$ is independent of $t$ equation 22, can be rewritten in the form

$$
\left(1+\lambda_{1}^{\alpha} D_{t}^{\alpha}\right) \frac{\partial p}{\partial x}=\left(1+\lambda_{2}^{\beta} \frac{t^{-\beta}}{\Gamma(1-\beta)}\right) A(x) .
$$

Here we have used the identity

$$
D_{t}^{\beta} 1=\frac{t^{-\beta}}{\Gamma(1-\beta)}, \quad 0<\beta<1,
$$

obtained by applying the definition (51) of the Riemann-Liouville fractional derivative and identity (50). Eq. (24) implies that the pressure gradient can be expressed as follows

$$
\frac{\partial p}{\partial x}=A(x) y(t)
$$

where the function $y(t)$ is a solution of the equation

$$
\left(1+\lambda_{1}^{\alpha} D_{t}^{\alpha}\right) y(t)=1+\lambda_{2}^{\beta} \frac{t^{-\beta}}{\Gamma(1-\beta)} .
$$

Therefore, the time evolution of the pressure gradient is determined by the behavior of the function $y(t)$. In the present work our study is limited to the properties of this function.

In what follows we assume $\lambda_{1} \neq 0$. Let us rewrite (27) in the form of the following fractional order equation

$$
D_{t}^{\alpha} y(t)=-\frac{1}{\lambda_{1}^{\alpha}} y(t)+F(t),
$$


E. Bazhlekova et al., Peristaltic transport of viscoelastic bio-fluids ...

where

$$
F(t)=\frac{1}{\lambda_{1}^{\alpha}}\left(1+\lambda_{2}^{\beta} \frac{t^{-\beta}}{\Gamma(1-\beta)}\right) .
$$

Suppose the physically reasonable initial condition

$$
\left.\frac{\partial p}{\partial x}\right|_{t=0}<\infty
$$

Therefore, from 26, $y(0)<\infty$ and thus

$$
\lim _{t \rightarrow 0}\left(J_{t}^{1-\alpha} y\right)(t)=0
$$

where $J_{t}^{1-\alpha}$ is a Riemann-Liouville fractional integral, see 47) in the Appendix. According to 63. the solution of equation (28) is given by

$$
y(t)=\int_{0}^{t} \tau^{\alpha-1} E_{\alpha, \alpha}\left(-\frac{\tau^{\alpha}}{\lambda_{1}^{\alpha}}\right) F(t-\tau) \mathrm{d} \tau,
$$

where $E_{\alpha, \alpha}(\cdot)$ denotes the Mittag-Leffler function (see (55) for the definition). From (32), 29) and the definition (47) of the fractional integration operator we deduce the representation

$$
y(t)=\frac{1}{\lambda_{1}^{\alpha}}\left(J_{t}^{1}+\lambda_{2}^{\beta} J_{t}^{1-\beta}\right)\left(t^{\alpha-1} E_{\alpha, \alpha}\left(-\frac{t^{\alpha}}{\lambda_{1}^{\alpha}}\right)\right),
$$

which by the use of identities (61) and 59) implies that the function $y(t)$ can be expressed in terms of the Mittag-Leffler functions as follows

$$
\begin{aligned}
y(t) & =1-E_{\alpha, 1}\left(-\frac{t^{\alpha}}{\lambda_{1}^{\alpha}}\right) \\
& +\frac{\lambda_{2}^{\beta}}{\lambda_{1}^{\alpha}} t^{\alpha-\beta} E_{\alpha, \alpha+1-\beta}\left(-\frac{t^{\alpha}}{\lambda_{1}^{\alpha}}\right) .
\end{aligned}
$$

Inserting (55) into 33) one obtains the following series expansion

$$
\begin{aligned}
y(t) & =1-\sum_{k=0}^{\infty} \frac{(-1)^{k} t^{\alpha k}}{\lambda_{1}^{\alpha k} \Gamma(\alpha k+1)} \\
& +\lambda_{2}^{\beta} \sum_{k=0}^{\infty} \frac{(-1)^{k} t^{\alpha k+\alpha-\beta}}{\lambda_{1}^{\alpha(k+1)} \Gamma(\alpha k+\alpha-\beta+1)} .
\end{aligned}
$$

In the particular case $\alpha=\beta$ (33) reduces to

$$
y(t)=1-\left(1-\left(\frac{\lambda_{2}}{\lambda_{1}}\right)^{\alpha}\right) E_{\alpha, 1}\left(-\frac{t^{\alpha}}{\lambda_{1}^{\alpha}}\right),
$$

while for $\lambda_{2}=0$ it gives

$$
y(t)=1-E_{\alpha, 1}\left(-\frac{t^{\alpha}}{\lambda_{1}^{\alpha}}\right) .
$$

Based on the obtained expressions we study the behavior of the function $y(t)$. Recall the restrictions on the parameters $\lambda_{1} \geq \lambda_{2} \geq 0,0<\alpha \leq 1$ and $0<\beta \leq 1$.

In the simplest case $\lambda_{2}=0$, based on representation (36) and the properties of MittagLeffler function, we easily infer that $y(t)$ is a monotonically increasing function with $y(0)=0$ and $y(+\infty)=1$. Moreover, for small times $t$ the function $y(t)$ grows faster when $\alpha$ is smaller, while for large $t$ it grows faster (and approaches the value 1 ) when $\alpha$ is larger. This behavior can be seen also on Fig. 1 .

Qualitatively similar behavior can be deduced from the representation (35) for the case $\alpha=\beta$, taking into account that $\lambda_{1} \geq \lambda_{2}$ (see also Fig. 5 and Fig. 6. However, there is one essential difference: in this case $y(t)$ does not vanish at $t=0$, more precisely, (35) implies

$$
y(0)=\left(\frac{\lambda_{2}}{\lambda_{1}}\right)^{\alpha}, \quad \alpha=\beta .
$$

To find the asymptotic behavior of $y(t)$ for $t \rightarrow$ 0 we take the first terms in the series representation (34) and obtain for $\lambda_{2} \neq 0$ :

$$
y(t)=\frac{\lambda_{2}^{\beta}}{\lambda_{1}^{\alpha}} \frac{t^{\alpha-\beta}}{\Gamma(1+\alpha-\beta)}+O\left(t^{\min \{\alpha, 2 \alpha-\beta\}}\right),
$$

and for $\lambda_{2}=0$ :

$$
y(t)=\frac{1}{\lambda_{1}^{\alpha}} \frac{t^{\alpha}}{\Gamma(1+\alpha)}+O\left(t^{2 \alpha}\right) .
$$

Therefore, for $\lambda_{2}=0$ as well as for $\lambda_{2} \neq 0$ and $\alpha>\beta$ the function $y(t)$ vanishes for $t \rightarrow 0$. If $\lambda_{2} \neq 0$ and $\alpha=\beta$ then the initial value $y(0)$ is as in (37).

However, if $\lambda_{2} \neq 0$ and $\alpha<\beta$, then the asymptotic expansion (38) implies that the function $y(t)$ has a weak singularity as $t \rightarrow 0$ (see also Fig. 3). This contradicts initial condition (30) and raises the question whether the model is physically correct in this case. 
Concerning large time behavior, asymptotic expansion (56) in the Appendix implies

$$
\begin{aligned}
y(t) & =1-\lambda_{1}^{\alpha} \frac{t^{-\alpha}}{\Gamma(1-\alpha)}+\lambda_{2}^{\alpha} \frac{t^{-\beta}}{\Gamma(1-\beta)} \\
& +O\left(t^{-\min \{\alpha+\beta, 2 \alpha\}}\right), t \rightarrow+\infty .
\end{aligned}
$$

This asymptotic expansion is valid in all of the considered cases. Therefore, in all cases

$$
\lim _{t \rightarrow+\infty} y(t)=1 \text {. }
$$

This can also be observed on the figures.

\section{NUMERICAL METHOD}

Let us note first that the explicit representation (34) derived from the series expansions of the Mittag-Leffler functions is appropriate for numerical computation of the function $y(t)$ only for sufficiently small times.

In [24], [25], [26], [27] two semi-numerical techniques are used for the solution of Eq. (27): Adomian decomposition method (ADM) and homotopy analysis method (HAM). These two methods give a series of functions, which first terms are used for the numerical computation of $y(t)$. It appears that the obtained approximations of $y(t)$ by these two methods (for the chosen parameters in HAM $\hbar=-1$ and $p_{0}=0$ ) are the same as if we take the first terms of the series in (34). Therefore, it can be expected that the numerical techniques proposed in these studies retain the aforementioned disadvantage of using the series expansion 34 for numerical computation: they work only for sufficiently small times. In contrast, the numerical technique used in the present work is appropriate for all times.

For numerical computation of the function $y(t)$ we use an algorithm based on its representation as a solution of an integral equation. Applying the operator $J_{t}^{\alpha}$ to both sides of equation (28) and using (31), (52), and the semi-group property (49), we obtain that $y(t)$ satisfies the following integral equation

$$
y(t)=-\frac{1}{\lambda_{1}^{\alpha}} \int_{0}^{t} \frac{(t-\tau)^{\alpha-1}}{\Gamma(\alpha)} y(\tau) \mathrm{d} \tau+H(t),
$$

where

$$
H(t)=\frac{1}{\lambda_{1}^{\alpha}}\left(\frac{t^{\alpha}}{\Gamma(\alpha+1)}+\lambda_{2}^{\beta} \frac{t^{\alpha-\beta}}{\Gamma(\alpha-\beta+1)}\right) .
$$

Equation 42 is used here for the numerical computation of the function $y(t)$, applying the socalled fractional Adams method, originally proposed and analyzed by Diethelm et al. [6], [7]. This is a predictor-corrector method in which as predictor the fractional Adams-Bashforth method is used and as corrector the fractional AdamsMoulton method. For completeness, here we give the numerical scheme.

To find a numerical solution of Eq. (42) in the time interval $t \in[0, T]$ consider a uniform grid $\left\{t_{j}=j h, j=0,1, \ldots, N\right\}$ with some integer $N$ and $h=T / N$. Denote by $y_{j}$ the approximation for $y\left(t_{j}\right)$. For the sake of brevity the notation $\lambda=$ $-\lambda_{1}^{-\alpha}$ is used.

The predictor $y_{k+1}^{P}$ is determined by the formula

$$
y_{k+1}^{P}=\frac{\lambda}{\Gamma(\alpha)} \sum_{j=0}^{k} b_{j, k+1} y_{j}+H\left(t_{k}\right),
$$

where

$$
b_{j, k+1}=\frac{h^{\alpha}}{\alpha}\left((k+1-j)^{\alpha}-(k-j)^{\alpha}\right) .
$$

The corrector formula is given by

$$
\begin{aligned}
y_{k+1} & =\frac{\lambda}{\Gamma(\alpha)}\left(\sum_{j=0}^{k} a_{j, k+1} y_{j}+a_{k+1, k+1} y_{k+1}^{P}\right) \\
& +H\left(t_{k+1}\right)
\end{aligned}
$$

where

$$
a_{j, k+1}=\frac{h^{\alpha}}{\alpha(\alpha+1)} A_{j, k+1}
$$

and $A_{j, k+1}$ are defined by

$$
A_{j, k+1}=\left\{\begin{array}{cl}
k^{\alpha+1}-(k-\alpha)(k+1)^{\alpha} & \text { if } j=0, \\
(k-j+2)^{\alpha+1}+(k-j)^{\alpha+1} & \text { if } 1 \leq j \leq k, \\
-2(k-j+1)^{\alpha+1} & \text { if } j=k+1 .
\end{array}\right.
$$

Using this numerical algorithm, the function $y(t)$ is computed for several values of the parameters. The performed numerical experiments indicate 
that this method is fast and stable. Although, due to the calculation of the integral, it is more time consuming for larger $T$, the numerical experiments with $T=10$ and $T=100$ indicate that the method works sufficiently well also for large time intervals.

\section{NUMERICAL RESULTS AND DISCUSSION}

In this section we discuss some results for the function $y(t)$, obtained by the numerical technique described above. Recall that the graphs of $y(t)$ represent the time evolution of the pressure gradient.

On Fig. 1 and Fig. 2 plots of the function $y(t)$ are presented for $\lambda_{2}=0$, which corresponds to the case of fractional Maxwell model, considered in [24]. Comparing these two figures to [24], Fig. 1 and Fig. 2, we observe the same behavior (for better comparison we have chosen the same values for the parameter $\alpha$ as in [24]). The time profiles on Fig. 1 exhibit increasing pressure gradient with time as for smaller $\alpha$ it increases faster for small $t$ and slower for large $t$, whereas for larger $\alpha$ the situation is opposite: it increases slower for small $t$ and faster for large $t$. This is in agreement with the theoretical observations in Section III based on the analytical representation (36). Unlike the figures in [24], where only the time interval $t \in[0,1]$ is considered, on Fig. 1 we also give plots for $t>1$, which reveal that the pressure gradient does not grow infinitely with time and approaches a certain value $(A(x))$. This confirms the theoretical observations in Section III. On Fig. 2, where the influence of the relaxation time $\lambda_{1}$ is illustrated, we see that the pressure gradient is smaller for larger values of $\lambda_{1}$. Therefore this parameter resists the movement of the flow.

Figures 3-6 correspond to the general case $\lambda_{2} \neq$ 0 .

On Fig. 3 the behavior of the pressure gradient function for $\alpha<\beta$ is illustrated. It is seen that the pressure gradient has a singularity at $t=0$ $(y(0)=+\infty)$. This was also observed in Section III. To the best of our knowledge, this feature of the model has not been discussed before and raises the question of its physical adequacy. In the works
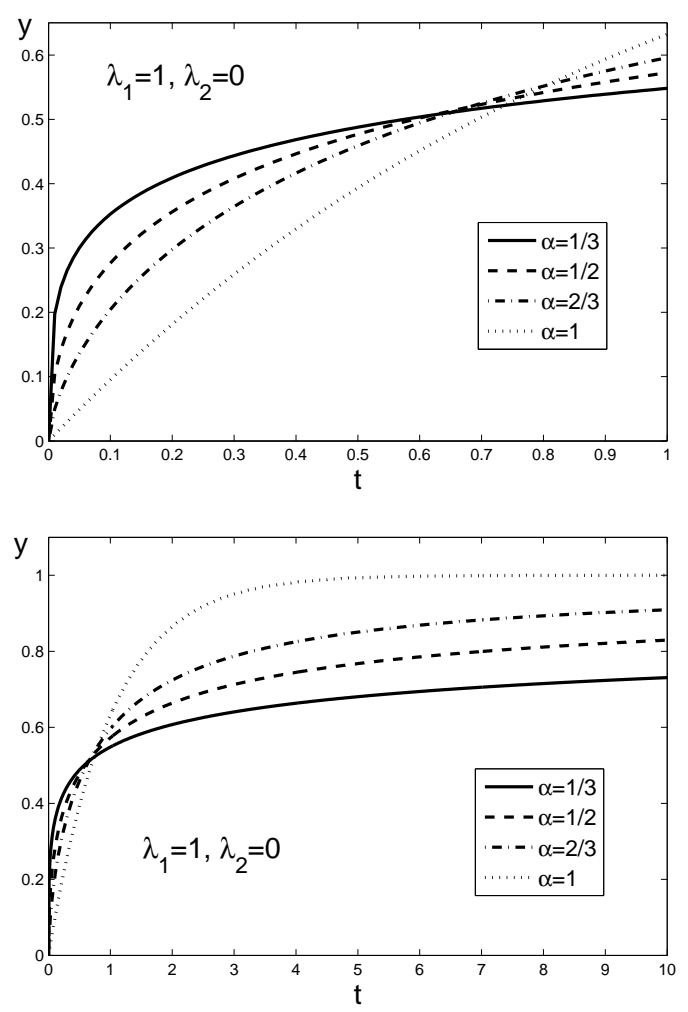

Fig. 1. Time profile of the pressure gradient for $A=1$, $\lambda_{1}=1, \lambda_{2}=0$, and various values of $\alpha$. Time interval $[0,1]$ (above) and $[0,10]$ (below).

[26] and [27], where the case $\alpha \leq \beta$ is considered, this issue has not been addressed.

On Fig. 4 the case $\alpha>\beta$ is illustrated. Comparing Fig. 3 and Fig. 4 it is seen that the behavior for $\alpha>\beta$ is qualitatively different from those for $\alpha<\beta$. For small times the pressure gradient is monotonically decreasing for $\alpha<\beta$ (Fig. 3) and monotonically increasing for $\alpha>\beta$ (Fig. 4). However, this monotonic behavior is not retained for all $t$. The influence of the fractional parameters $\alpha$ and $\beta$ observed on both figures is as follows. The effect of the fractional parameter $\alpha$ for small times is opposite to that for large times. The same holds for the fractional parameter $\beta$. In addition, the effects of the parameters $\alpha$ and $\beta$ are found to be opposite to each other.

Plots for the case $\alpha=\beta$ are given on Fig. 5 and Fig. 6. The influence of the relaxation time 


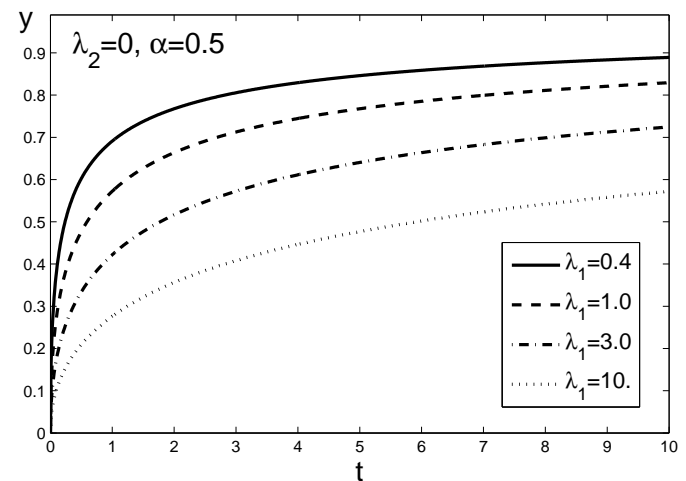

Fig. 2. Time profile of the pressure gradient for $A=1$, $\lambda_{2}=0, \alpha=0.5$ and various values of $\lambda_{1}$.
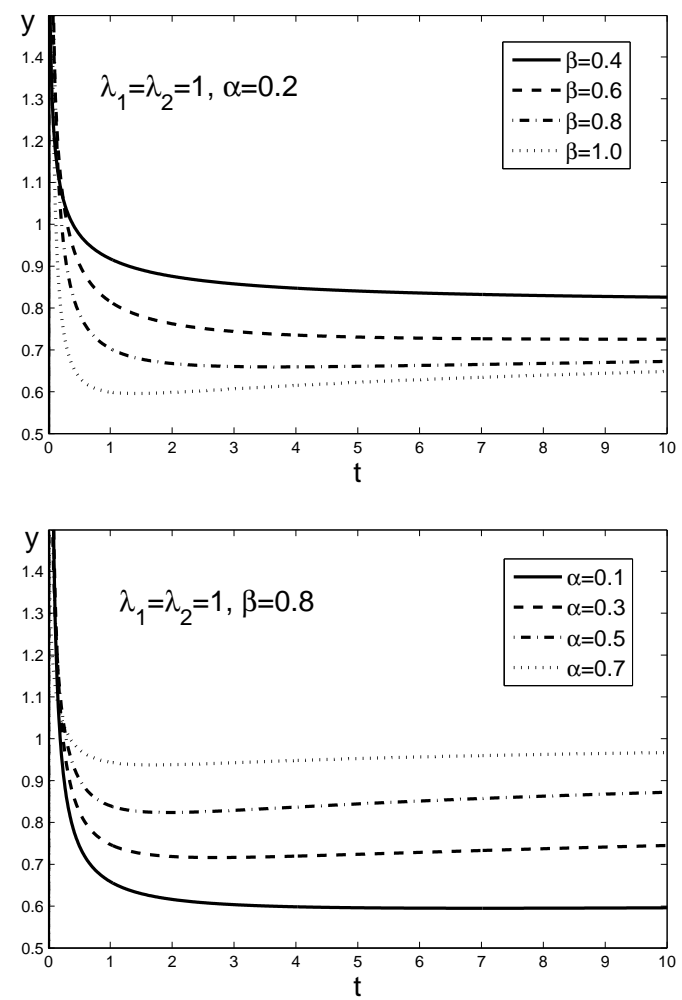

Fig. 3. Time profiles of the pressure gradient for $\alpha<\beta$, $A=1$ and $\lambda_{1}=\lambda_{2}=1$.

$\lambda_{1}$ and retardation time $\lambda_{2}$ observed on Fig. 5 is as follows. The pressure gradient increases with the retardation time $\lambda_{2}$ whereas it decreases with the relaxation time $\lambda_{1}$. On Fig. 6 the effect of
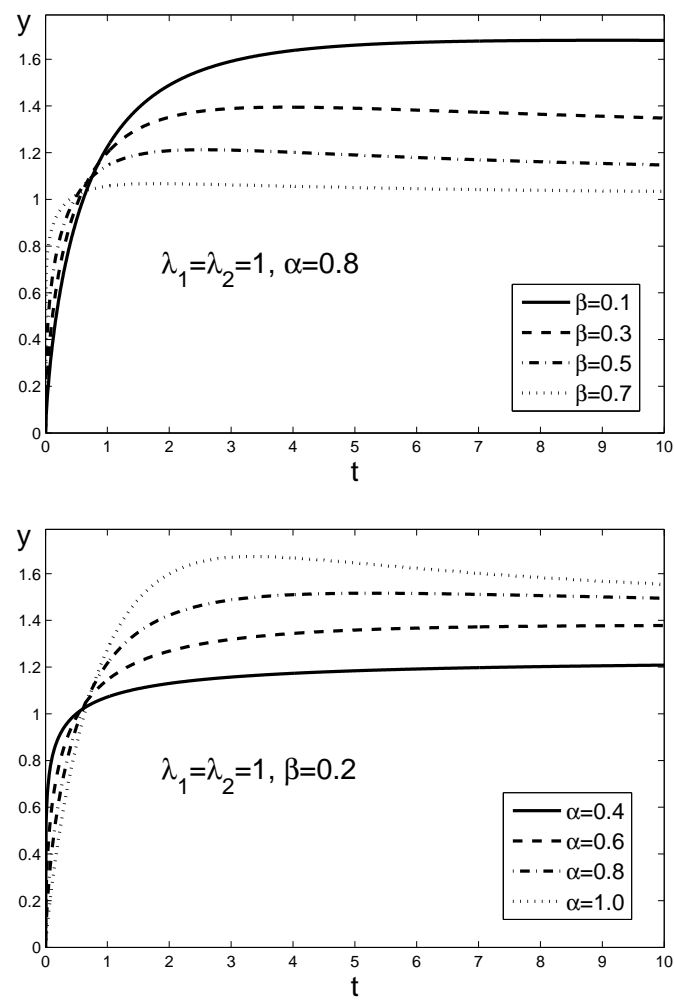

Fig. 4. Time profiles of the pressure gradient for $\alpha>\beta$, $A=1$ and $\lambda_{1}=\lambda_{2}=1$.

the fractional parameter $\alpha$ is examined. In order to capture the peculiarities of the function $y(t)$ a larger time interval is considered $t \in[0,100]$. The influence of the fractional parameter resemble those observed on Fig. 1 in the case of fractional Maxwell model. This is in agreement with the similarity in the explicit expressions (35) and (36).

\section{CONCLUSIONS}

Employing the mathematical tools of Fractional Calculus we study in this work the time evolution of the pressure gradient in a viscoelastic peristaltic flow with fractional Oldroyd-B constitutive model. The analysis of the effect of different parameters shows that for $\alpha<\beta$ there is an unphysical singularity. This means that from the previously considered in [26] and [27] range $0<\alpha \leq$ $\beta \leq 1$ only for $\alpha=\beta$ the model is physically meaningful. This is also in agreement with the 

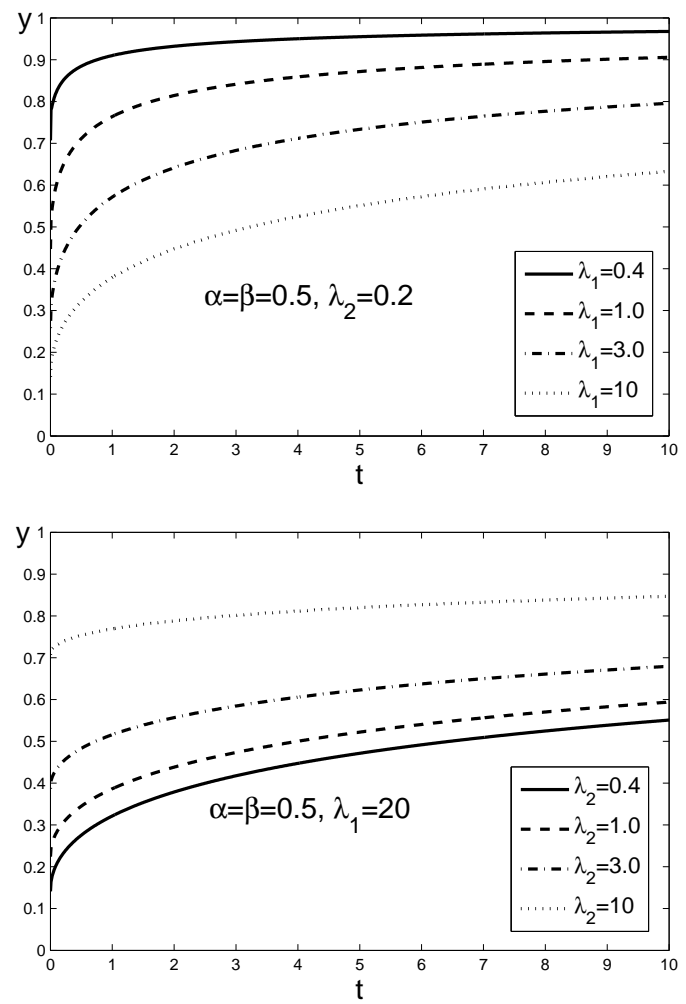

Fig. 5. Time profiles of the pressure gradient for $\alpha=\beta=$ $0.5, A=1$ and various values of $\lambda_{1}$ and $\lambda_{2}, \lambda_{1}>\lambda_{2}$.

statement in [30], that the Oldroyd-B constitutive law is thermodynamically compatible only if the fractional orders $\alpha$ and $\beta$ coincide and $\lambda_{1} \geq \lambda_{2}$.

In both cases of physical interest: $\lambda_{2}=0$, $0<\alpha \leq 1$ (fractional Maxwell model) and $\lambda_{1} \geq \lambda_{2}>0,0<\alpha=\beta \leq 1$ (thermodynamically compatible Oldroyd-B model) the pressure gradient across one wavelength is monotonically increasing with time and approaches a certain stationary value. The same qualitative behavior will hold for the pressure rise and friction force.

The technique used in this work can be applied to more general fractional derivative viscoelastic models of peristaltic transport, such as models with more complicated geometry (non-uniform, cylindrical, inclined channels), flows with slip effects, flows in porous media, etc.
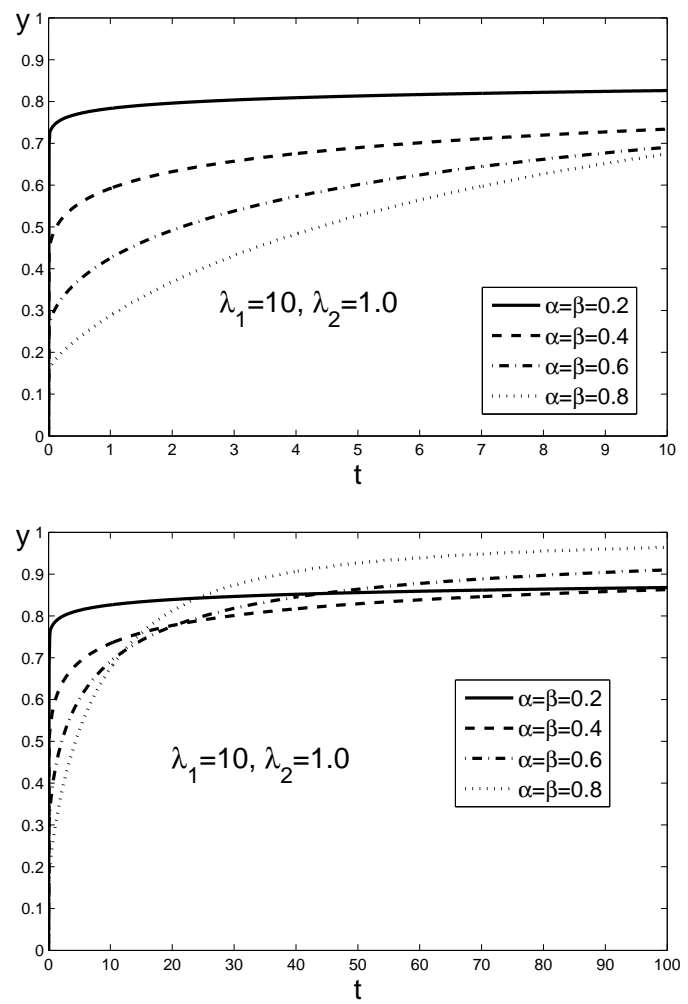

Fig. 6. Time profiles of the pressure gradient for $\alpha=\beta$, $A=1, \lambda_{1}=10, \lambda_{2}=1$. Time interval $[0,10]$ (above) and $[0,100]$ (below).

\section{ACKNOWLEDGMENTS}

The work is partially supported by Grant DFNI-I02/9 from the Bulgarian National Science Fund; and the bilateral research project between Bulgarian and Serbian academies of sciences (2014-2016) "Mathematical modeling via integraltransform methods, partial differential equations, special and generalized functions, numerical analysis".

\section{APPENDIX}

Here we summarize some facts from Fractional Calculus, for details see [12], [16].

The fractional order Riemann-Liouville integral $J_{t}^{\alpha}$ is defined by

$$
J_{t}^{\alpha} f(t)=\int_{0}^{t} \omega_{\alpha}(t-\tau) f(\tau) \mathrm{d} \tau, \quad \alpha>0
$$


E. Bazhlekova et al., Peristaltic transport of viscoelastic bio-fluids ...

where

$$
\omega_{\alpha}(t)=\frac{t^{\alpha-1}}{\Gamma(\alpha)}, \quad \alpha>0, t>0 .
$$

Here $\Gamma(\cdot)$ is the Gamma function. Basic properties of this function are $\Gamma(1)=1, \Gamma(\alpha+1)=\alpha \Gamma(\alpha)$. Therefore $\omega_{1}(t) \equiv 1$.

The operators of fractional integration satisfy the semi-group property:

$$
J_{t}^{\alpha} J_{t}^{\beta}=J_{t}^{\alpha+\beta}, \quad \alpha, \beta>0,
$$

or, equivalently,

$$
J_{t}^{\alpha} \omega_{\beta}=\omega_{\alpha+\beta}, \quad \alpha, \beta>0 .
$$

The Riemann-Liouville fractional derivative $D_{t}^{\alpha}$ of order $\alpha \in(0,1]$ is defined by $D_{t}^{1}=\mathrm{d} / \mathrm{d} t$ and

$$
D_{t}^{\alpha}=D_{t}^{1} J_{t}^{1-\alpha}
$$

The Riemann-Liouville fractional derivatives and integrals are related via the identities:

$$
D_{t}^{\alpha} J_{t}^{\alpha} f=f, \quad \alpha>0,
$$

and

$$
J_{t}^{\alpha} D_{t}^{\alpha} f=f-\left(J_{t}^{1-\alpha} f\right)(0) \omega_{\alpha}(t), \alpha \in(0,1) .
$$

Application of the Laplace transform

$$
\mathcal{L}\{f(t)\}(s)=\widehat{f}(s)=\int_{0}^{\infty} e^{-s t} f(t) d t
$$

to the operator of fractional integration gives

$$
\mathcal{L}\left\{J_{t}^{\alpha} f\right\}(s)=s^{-\alpha} \widehat{f}(s), \quad \alpha>0,
$$

which implies the following identity for the Riemann-Liouville fractional derivative of order $\alpha \in(0,1)$ :

$$
\mathcal{L}\left\{D_{t}^{\alpha} f\right\}(s)=s^{\alpha} \widehat{f}(s)-\left(J_{t}^{1-\alpha} f\right)(0) .
$$

Denote as usual by $E_{\alpha, \beta}(\cdot)$ the two-parameter Mittag-Leffler function

$$
E_{\alpha, \beta}(z)=\sum_{k=0}^{\infty} \frac{z^{k}}{\Gamma(\alpha k+\beta)} .
$$

For $\alpha \in(0,2), \beta>0$, the Mittag-Leffler function has the following asymptotic expansion as $t \rightarrow$ $+\infty$

$$
E_{\alpha, \beta}(-t)=-\sum_{k=1}^{N-1} \frac{(-t)^{-k}}{\Gamma(\beta-\alpha k)}+O\left(t^{-N}\right) .
$$

An important particular case is

$$
E_{1,1}(-t)=\exp (-t)
$$

and some properties of the function $E_{\alpha, 1}(-t)$ for $0<\alpha<1$ resemble the behavior of the exponential function: $E_{\alpha, 1}(-t)$ is monotonically decreasing with $E_{\alpha, 1}(0)=1, E_{\alpha, 1}(-\infty)=0$. However, unlike the fast exponential decay of $\exp (-t)$ for large $t$, the Mittag-Leffler function admits a slow algebraic decay, which is slower for smaller $\alpha$. At $t=0$ the opposite picture is observed: the Mittag-Leffler function admits a fast decay $\left(\frac{\mathrm{d}}{\mathrm{d} t} E_{\alpha, 1}(-t) \rightarrow \infty\right.$ for $t \rightarrow 0$, see 600$)$, and this decay is faster for smaller $\alpha$.

Recall the Laplace transform pairs

$$
\begin{aligned}
\mathcal{L}\left\{\omega_{\alpha}(t)\right\}(s) & =s^{-\alpha} \\
\mathcal{L}\left\{t^{\beta-1} E_{\alpha, \beta}\left(\lambda t^{\alpha}\right)\right\}(s) & =\frac{s^{\alpha-\beta}}{s^{\alpha}-\lambda} .
\end{aligned}
$$

The following identity is often useful

$$
J_{t}^{\gamma}\left(t^{\beta-1} E_{\alpha, \beta}\left(\lambda t^{\alpha}\right)\right)=t^{\beta+\gamma-1} E_{\alpha, \beta+\gamma}\left(\lambda t^{\alpha}\right),
$$

where $\alpha, \beta, \gamma, t>0$. It can be proven by applying Laplace transform and using (53) and (58).

Another useful property is the following

$$
\frac{\mathrm{d}}{\mathrm{d} t} E_{\alpha, 1}\left(\lambda t^{\alpha}\right)=\lambda t^{\alpha-1} E_{\alpha, \alpha}\left(\lambda t^{\alpha}\right) .
$$

It can be deduced again by applying Laplace transform and using (58) or directly from the series representation (55) of the Mittag-Leffler function. Integrating 60 and using that $E_{\alpha, 1}(0)=1$ we obtain for $\alpha>0$ and $t>0$

$$
J_{t}^{1}\left(t^{\alpha-1} E_{\alpha, \alpha}\left(\lambda t^{\alpha}\right)\right)=-\frac{1}{\lambda}\left(1-E_{\alpha, 1}\left(\lambda t^{\alpha}\right)\right)
$$

Let $f(t)$ be an integrable on $(0, T)$ function and let $\alpha \in(0,1)$. Then the differential equation of fractional order

$$
D_{t}^{\alpha} y(t)=\lambda y(t)+f(t), \quad t>0,
$$


has a unique solution given by

$$
\begin{aligned}
y(t) & =y_{0} t^{\alpha-1} E_{\alpha, \alpha}\left(\lambda t^{\alpha}\right) \\
& +\int_{0}^{t} \tau^{\alpha-1} E_{\alpha, \alpha}\left(\lambda \tau^{\alpha}\right) f(t-\tau) \mathrm{d} \tau,
\end{aligned}
$$

where $y_{0}=\lim _{t \rightarrow 0} J_{t}^{1-\alpha} y$. This result can be found in [16], p. 137. The easiest way to prove it is by applying Laplace transform.

\section{REFERENCES}

[1] N.S. Akbar, M. Raza, R. Ellahi, Peristaltic flow with thermal conductivity of $\mathrm{H}_{2} \mathrm{O}+\mathrm{Cu}$ nanofluid and entropy generation, Results Phys. 5 (2015) 115-124. http://dx.doi.org/10.1016/j.rinp.2015.04.003

[2] N.S. Akbar, M. Raza, R. Ellahi, Influence of induced magnetic field and heat flux with the suspension of carbon nanotubes for the peristaltic flow in a permeable channel, J Magn Magn Mater. 38 (2015) 405-415. http://dx.doi.org/10.1016/j.jmmm.2014.12.087

[3] T.M. Atanacković, S. Pilipović, B. Stanković, D. Zorica, Fractional Calculus with Applications in Mechanics: Vibrations and Diffusion Processes, John Wiley \& Sons, 2014.

[4] R.L. Bagley, P.J. Torvik, On the fractional calculus model of viscoelastic behavior, J Rheol. 301 (1986) 133-155. http://dx.doi.org/10.1122/1.549887

[5] E. Bazhlekova, I. Bazhlekov, Viscoelastic flows with fractional derivative models: computational approach via convolutional calculus of Dimovski, Fract Calc Appl Anal. 174 (2014) 954-976. http://dx.doi.org/10.2478/s13540-014-0209-x

[6] K. Diethelm, N.J. Ford, A.D. Freed, Detailed error analysis for a fractional Adams method, Numer Algorithms 361 (2004) 31-52. http://dx.doi.org/10.1023/B:NUMA.0000027736.85078. be

[7] K. Diethelm, N.J. Ford, A.D. Freed, A predictor-corrector approach for the numerical solution of fractional differential equations, Nonlinear Dynam. 291 (2002) 3-22. http://dx.doi.org/10.1023/A:1016592219341

[8] R. Ellahi, F. Hussain, Simultaneous effects of MHD and partial slip on peristaltic flow of Jeffery fluid in a rectangular duct, J Magn Magn Mater. 393 (2015) 284292. http://dx.doi.org/10.1016/j.jmmm.2015.05.071

[9] R. Ellahi, S.U. Rahman, S. Nadeem, K. Vafai, The blood flow of Prandtl fluid through a tapered stenosed arteries in permeable walls with magnetic field, Commun Theor Phys. 6303 (2015) 353-358. http://dx.doi.org/10.1088/0253-6102/63/3/353

[10] M. Fabrizio, Fractional rheological models for thermomechanical systems. Dissipation and free energies, Fract Calc Appl Anal. 171 (2014) 206-223. http://dx.doi.org/10.2478/s13540-014-0163-7
[11] C. Fetecau, M. Jamil, C. Fetecau, D. Vieru, The Rayleigh-Stokes problem for an edge in a generalized Oldroyd-B fluid, Z Angew Math Phys. 605 (2009), 921933.

[12] R. Gorenflo, F. Mainardi, Fractional calculus: integral and differential equations of fractional order. In: A. Carpinteri, F. Mainardi (Eds.), Fractals and Fractional Calculus in Continuum Mechanics, SpringerVerlag, Wien/New York (1997) 223-276.

[13] J. Hristov, Integral-balance solution to the Stokes first problem of a viscoelastic generalized second grade fluid, Therm Sci. 16 (2012), 395-410. http://dx.doi.org/10.2298/TSCI110401077H

[14] M. Jaffrin, A. Shapiro, Peristaltic pumping, Annu Rev Fluid Mech. 3 (1971) 13-36. http://dx.doi.org/10.1146/annurev.fl.03.010171.000305

[15] M.S. Kandelousi, R. Ellahi, Simulation of ferrofluid flow for magnetic drug targeting using Lattice Boltzmann method, Z Naturforsch. A 70 (2015) 115-124. http://dx.doi.org/10.1515/zna-2014-0258

[16] A.A. Kilbas, H.M. Srivastava, J.J. Trujillo, Theory and applications of fractional differential equations, North-Holland Mathematics studies, Amsterdam: Elsevier, 2006.

[17] Y. Liu, L. Zheng, X. Zhang, Unsteady MHD Couette flow of a generalized Oldroyd-B fluid with fractional derivative, Comput Math Appl. 612 (2011) 443-450. http://dx.doi.org/10.1016/j.camwa.2010.11.021

[18] F. Mainardi, Fractional Calculus and Waves in Linear Viscoelasticity, London: Imperial College Press, 2010.

[19] F. Mainardi, An historical perspective on fractional calculus in linear viscoelasticity, Fract Calc Appl Anal. 154 (2012), 712-717. http://dx.doi.org/10.2478/s13540-012-0048-6

[20] S.P. Näsholm, S. Holm, On a fractional Zener elastic wave equation, Fract Calc Appl Anal. 161 (2013) 2650. http://dx.doi.org/10.2478/s13540-013--0003-1

[21] H. Qi, M. Xu, Some unsteady unidirectional flows of a generalized Oldroyd-B fluid with fractional derivative, Appl Math Model. 33 (2009) 4184-4191. http://dx.doi.org/10.1016/j.apm.2009.03.002

[22] S. Rogosin, F. Mainardi, George William Scott Blair the pioneer of fractional calculus in rheology, Commun Appl Ind Math. 61 (2014) e481. http://dx.doi.org/10.1685/journal.caim.481

[23] D.Y. Song, T.Q. Jiang, Study on the constitutive equation with fractional derivative for the viscoelastic fluids Modified Jeffreys model and its application, Rheol Acta 37 (1998) 512-517. http://dx.doi.org/10.1007/s003970050138

[24] D. Tripathi, S.K. Pandey, S. Das, Peristaltic flow of viscoelastic fluid with fractional Maxwell model through a channel, Appl Math Comput. 215 (2010) 3645-3654. http://dx.doi.org/10.1016/j.amc.2009.11.002

[25] D. Tripathi, Peristaltic transport of fractional Maxwell fluids in uniform tubes: Applications in endoscopy, Com- 
E. Bazhlekova et al., Peristaltic transport of viscoelastic bio-fluids ...

put Math Appl. 623 (2011) 1116-1126.

http://dx.doi.org/10.1016/j.camwa.2011.03.038

[26] D. Tripathi, A mathematical model for the peristaltic flow of chyme movement in small intestine, Math Biosci. 233 (2011) 90-97. http://dx.doi.org/10.1016/j.mbs.2011.06.007

[27] D. Tripathi, O. Anwar Bég, J. L. Curiel-Sosa, Homotopy semi-numerical simulation of peristaltic flow of generalised Oldroyd-B fluids with slip effects, Comput Methods Biomech Biomed Engin. 174 (2014) 433-442. http://dx.doi.org/10.1080/10255842.2012.688109

[28] K. Vafai, A.A. Khan, S. Sajjad, R. Ellahi, The study of peristaltic motion of third grade fluid under the effects of Hall current and heat transfer, Z Naturforsch. A $\mathbf{7 0} 4$ (2015) 281-293 http://dx.doi.org/10.1515/zna-2014-0330

[29] D. Valério, J. Tenreiro Machado, V. Kiryakova, Some pioneers of the applications of fractional calculus, Fract Calc Appl Anal. 172 (2014) 552-578. http://dx.doi.org/10.2478/s13540-014-0185-1

[30] P. Yang, K.Q. Zhu, Thermodynamic compatibility and mechanical analogue of the generalized Jeffreys and generalized Oldroyd-B fluids with fractional derivatives, Sci China-Phys Mech Astron. 544 (2011) 737-742.

http://dx.doi.org/10.1007/s11433-011-4271-7 